\title{
Auto-Tuned Induction Coil Conductivity Sensor for In-Vivo Human Tissue Measurements
}

\author{
J. Heller and J. R. Feldkamp \\ Kimberly-Clark Corporation, Corporate Research and Engineering \\ 2100 Winchester Road, Neenah, WI 54956, U.S.A
}

\begin{abstract}
Auto-tuned induction coil technology, based upon phase-locked loop circuitry (PLL), was developed and shown to be an effective tool for in-vivo measurement of electrical conductivity of human tissues. Because electrical contact is not required, several disadvantages of the electrode method for conductivity determination are avoided, such as electrode polarization and variable conductivity associated with the stratum corneum of the epidermis. Fixed frequency excitation is supplied to a parallel tuned RLC circuit, or "sensor", while bias applied to a varactor diode is automatically adjusted via PLL circuitry to maintain the RLC sensor at resonance. Since resonant impedance of a coil positioned near a conductive object is known to be frequency dependent, such an arrangement permits precise calibration of the sensor against a set of standard Potassium Chloride solutions. In our experiments, a two-layer spiral coil is used with upper and lower spiral arms staggered so as to reduce inter-winding coil capacitance. Preliminary in-vivo testing was done on the forearms of a single male subject as a prelude to more extensive use in a clinical setting. In that instance, electrical conductivity at the proximal volar forearm location was shown to depend on forearm elevation. Clinical studies using our prototype, as well as further consideration of the "elevation effect", are discussed in a companion paper.
\end{abstract}

Keywords: Induction coil, electrical conductivity, phase-locked-loop, human tissues, impedance, resonance

\section{INTRODUCTION}

$\mathrm{E}$ LECTRICAL CONDUCTIVITY of human tissues depends on a number of variables, including: tissue type, physiological state, in vivo or in vitro measurement, and measurement methodology. Method of measurement itself may vary according to excitation type (AC or DC), whether direct electrical contact is required, tissue volume sampled, specimen temperature and others. There are typically two strategies employed to make electrical conductivity measurements. The first involves direct electrical contact with body tissues via specialized electrodes which must make intimate contact with the surfaces of the tissues under consideration. This method is suitable over a broad range of excitation frequencies, from audio well into the RF (radio frequency) region. The second uses an induction coil to induce electrical currents known as eddy currents in the tissue of interest without making direct electrical contact. Resulting coil impedance changes are used to obtain conductivity information. This method is best pursued at frequencies exceeding about $1 \mathrm{MHz}$ but not so high that skin effects prohibit a desired degree of tissue penetration.

Exemplary of the first approach, Haemmerich et al. [1] measured in vivo electrical conductivity of hepatic tumors induced in rats to demonstrate that abnormal, or diseased, tissues exhibit different electrical properties than healthy tissues. Plunge-probe style conductivity cells of well-defined geometry were used to evaluate either healthy or diseased tissues in live rats. A possible difficulty with this sort of measurement is that introduction of the probe will cause tissue perforation and unavoidable damage. Furthermore, electrode polarization can complicate data interpretation, essential to yielding true conductivity values. Another class of less invasive conductivity measurements, still requiring direct electrical contact, is electrical impedance tomography (EIT), which involves attachment of multiple electrodes to the skin (Holder, [2]). In order for electrical current to pass into the body, it must first pass through skin. Given the variable nature of the epidermis, such as thickness, hydration level, and chemical composition, measurements may be difficult to interpret. EIT is presently used only as a research tool. A brief discussion of some of the issues associated with electrode-based conductivity measurement is provided by Arnold and Meeten [3].

The second approach, involving electromagnetic induction, has been used successfully by Guardo et al [4] to measure thoracic conductivity using a single induction coil. Their measurements demonstrated an ability of induction coil methods to sense volumetric changes of the heart while passing through the cardiac cycle. Hart et al [6] used induction coil conductivity measurements, together with models, to suggest that their technique could be useful for identification of the presence of edema in brain tissues. Netz et al [5] used multiple induction coils to demonstrate an ability to detect changes in brain impedance, possibly resulting from edema, though only with physical models that simulated the collection of fluids in the cranial cavity. Aside from biological applications, the induction coil method has been a favorite tool of material scientists to noninvasively determine electrical conductivity of newly discovered materials (Schnyders et al, [7]).

There are two potential problems that may be identified with an induction coil method. In cases where instrumentation is intended to be compact and portable, electronics typically relies upon some form of the venerable marginal oscillator to provide a measure of coil impedance changes (Probst et al, [8]). Upon placing the coil adjacent to the specimen of interest, the high dielectric constant of the tissue under test causes substantial changes in inter-winding 
coil capacitance (Harpen, [9]), with oscillation frequency altered in the process. Since electrical conductivity via induction coil is quadratically dependent on excitation frequency (Harpen, [10]), results using a marginal oscillator are likely to be ambiguous, unless some strategy is used to lock oscillator frequency. Another issue with induction coil methods concerns the closeness of approach to body tissues (Zaman et al, [11]). As is well known, the magnetic induction field in the vicinity of a simple loop coil (as an example) falls off with the third power of distance along the coil axis once our observation point is beyond one coil radius (Jackson, [12], p. 183). This can be used to advantage, however, when attempting to confine the measurement to a specific region of space - but generally, the gap between coil and specimen must be controlled to assure reproducibility.

\section{METHODS}

To address the issues described in the preceding section, an auto-tuned induction coil method has been pursued at Kimberly-Clark Corporation using a surface coil. Auto-tuning uses a phase-locked loop (PLL) approach to maintaining a parallel RLC (resistance, inductance, and capacitance) circuit at resonance while under fixed frequency excitation. The capacitance component of the RLC circuit is partly comprised of a varactor which can be automatically adjusted to compensate for the inevitable variations in inter-winding capacitance occurring due to specimen contact. A flat, twolayer spiral surface coil etched onto a standard printed circuit board (PCB) has been used in testing thus far, with the two spiral layers staggered relative to each other in order to reduce inter-winding capacitance and promote easier lock with PLL controls. Because of the flat nature of the coil and small size, it is relatively easy to reproduce the specimen-coil gap distance by attachment of a plastic film over the coil, typically $0.1 \mathrm{~mm}$ thick. A picture of the induction coil used in clinical testing is shown in figure $1-$ its diameter is about $3.5 \mathrm{~cm}$.

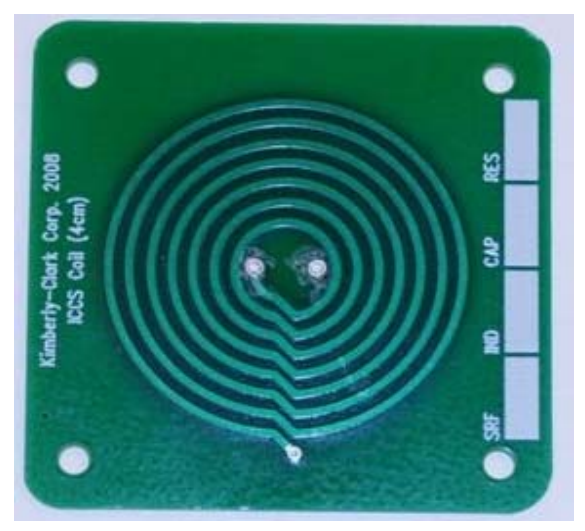

Fig.1 Photo of spiral coil along its main axis. A second foil, staggered from the first is unseen on the opposite side of the board. Diameter $=3.5 \mathrm{~cm}$.
An equivalent circuit of the induction coil [20], which captures the essential physics of its behavior, is shown in figure 2. Using an impedance analyzer to test several replicates of dual-layer spiral coils in free space, $\mathrm{R}_{\mathrm{s}}$ is nominally $0.37 \Omega \pm 0.02 \Omega, \mathrm{R}_{\mathrm{p}}$ is nominally about $27 \mathrm{k} \Omega \pm 0.5$ $\mathrm{k} \Omega, \mathrm{C}=9.67 \pm 0.03 \mathrm{pF}$ and $\mathrm{L}=3.35 \mu \mathrm{H} \pm 0.03 \mu \mathrm{H}$. In use, capacitance $\mathrm{C}$ was supplemented by additional parallel capacitance, in the form of a varactor diode, to permit autotuning, while $\mathrm{R}_{\mathrm{p}}$ was supplemented by additional parallel resistance to permit easier location of the resonant point (by reduction of circuit $\mathrm{Q}$ ).

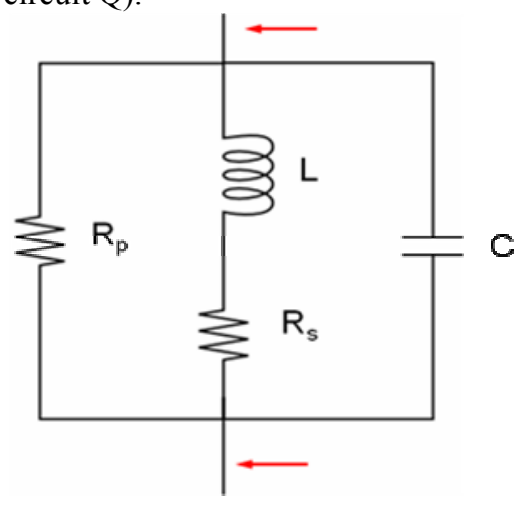

Fig.2 Equivalent circuit for an induction coil.

Referring to the circuit in figure 2 as the "sensor", sensor impedance and admittance at the resonant point of the sensor is easily derived as:

$$
A_{r e s}=\frac{1}{Z_{r e s}}=\frac{1}{R_{p}}+\frac{R_{s}}{R_{s}^{2}+X_{L}^{2}}
$$

Resonance is defined here as the frequency at which either admittance or impedance becomes purely real. Coil reactance is represented by its usual symbol, $X_{L}$. Provided RF frequencies exceed $10 \mathrm{MHz}$, then for a coil having inductance $L>$ about $3.0 \mu \mathrm{H}$ and series resistance $R_{s}<$ about $1 \Omega$, equation (1) can be approximated quite closely by:

$$
A_{\text {res }}=\frac{1}{Z_{\text {res }}} \cong \frac{1}{R_{p}}+\frac{R_{s}}{X_{L}^{2}}=\frac{1}{R_{p}}+\frac{R_{s}}{\omega^{2} L^{2}}
$$

Angular frequency is given by $\omega$. Series coil resistance $R_{s}$ arises from a combination of intrinsic coil resistance, which we call $R_{L}$, and a resistance $R_{E}$, originating in the eddy currents induced in any nearby conducting material, $R_{s}=R_{L}+R_{E}$. Furthermore, analytical efforts using Maxwell's equations to relate the real part of coil impedance, at resonance, to specimen conductivity (Harpen, [10], eq. 21) show that if frequency is not too large, the contribution to coil resistance coming from eddy currents varies linearly with specimen conductivity, $\sigma$, the square of angular frequency $\omega$, and the square of inductance: 


$$
R_{E}=\alpha^{\prime} \sigma \omega^{2} L^{2}
$$

Substituting (3) into (2) leads to a simple result, with $\alpha$ ' introduced as a frequency independent constant:

$$
A_{\text {res }}=\frac{1}{Z_{\text {res }}}=\left(\frac{1}{R_{p}}+\frac{R_{L}}{\omega^{2} L^{2}}\right)+\alpha^{\prime} \sigma
$$

The key feature of (4) is that admittance, $A_{\text {res }}$, ought to vary linearly with specimen conductivity, having slope that is independent of frequency, and an intercept that is nearly independent of frequency provided that $R_{p}$ is not too large. Recall that $R_{p}$ is deliberately reduced by placing additional resistance in parallel with the existing intrinsic coil resistance. With $\mathrm{R}_{\mathrm{L}}$ about $0.4 \Omega$ and $\mathrm{R}_{\mathrm{p}}$ set to about $20 \mathrm{k} \Omega$ by adding additional parallel resistance, the first term in parentheses in (4) is about an order of magnitude larger than the second, implying that intercept should be nearly frequency independent. Nevertheless, PLL electronics is used, in conjunction with varactor technology to maintain resonance at a fixed excitation frequency, which has the effect of forcing the intercept to be constant in practice. We note here that an alternative approach to PLL electronics is to sweep the capacitance of the varactor throughout some suitable range so that the RLC passes through resonance at the preset fixed excitation frequency. Using peak detection methods, determination of RLC impedance at resonance is again possible. Each method is accommodated in our prototypes, and has been used to "validate" the other.

\section{ICCS 2008 Prototype:}

Figure 3 provides a view of the handheld portable unit used to determine the resonant impedance, and thus admittance, of the sensor at fixed frequency.

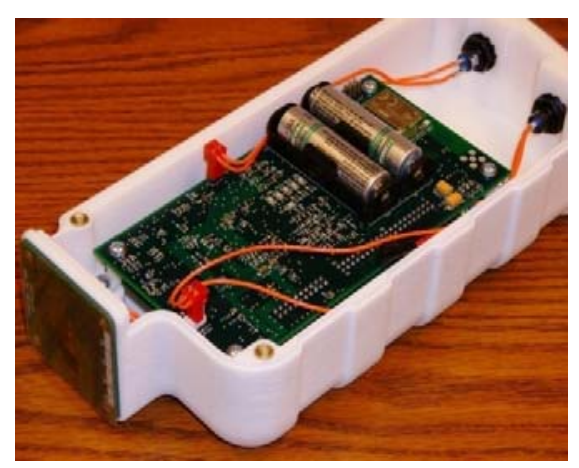

Fig.3 ICCS 2008 prototype with cover removed to show electronics (two stacked boards)

Because of the possible dependence of the intercept of (4) on frequency, though likely weak for our spiral coil, it is imperative that a fixed frequency be used for accurate measurements. In practice, the relative permittivity of the specimen is sufficiently high $(>>1)$ that inter-winding coil capacitance is expected to increase significantly upon placing the coil near to conductive tissues (Harpen, [9]), and thus detune the circuit - detuning has been observed to be as high as $0.5 \mathrm{MHz}$ in our own, strongly coupled measurements. Harpen [10] presents measurements, using simple loop coils loosely coupled to samples, showing about $0.1 \mathrm{MHz}$ of detuning when conductivity reaches about $4.5 \mathrm{~S} / \mathrm{m} \quad-$ dependence of detuning on conductivity was shown to be highly nonlinear (cubic).

Figure 4 provides a greatly simplified view, in block diagram form, of the electronics used to determine the resonant impedance, and thus admittance, of the sensor at fixed frequency. Maintaining resonance at a fixed excitation frequency is accomplished in either of two ways, though in either case, a "sense" resistor is placed in series with the circuit of figure 2 for the purpose of measuring voltage, current and phase angle (of voltage relative to current). In the PLL (phase locked loop) mode, phase angle is used to provide a DC feedback signal that is used to auto-tune a varactor placed in parallel with an induction coil, with the goal of maintaining phase angle nearly equal to zero, i.e. a resonant condition at the selected frequency.

The other mode, using swept capacitance, simply sweeps the capacitance range of the varactor while the applied RF excitation is kept at fixed frequency, forcing the sensor to "pass through" a resonant condition at the applied fixed frequency of the source. The latter mode determines the resonant point by noting when voltage has maximized. Though there are simpler induction coil methods to measure electrical conductivity, such as the marginal oscillator (L. Hart et. al., [6]), these do not typically provide a means to compensate for detuning.

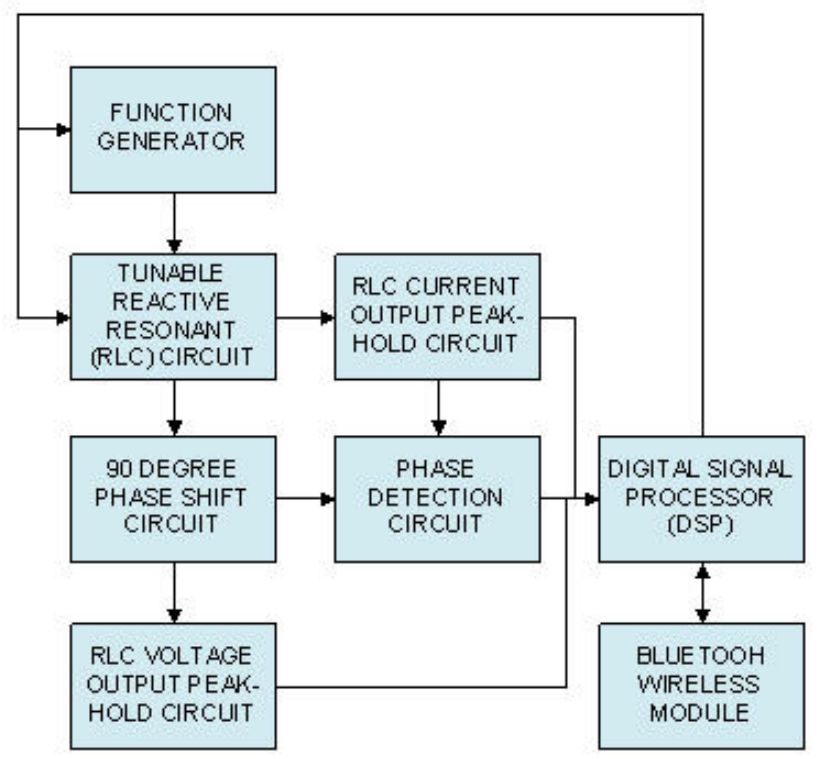

Fig.4 Block diagram of auto-tuned, resonant induction coil conductivity sensor. 


\section{A. Function Generator}

The purpose of the function generator is to provide a highly stable constant amplitude sinusoidal voltage to drive the tunable reactive resonant circuit. The heart of this circuit is the Analog Devices 9859 Direct Digital Synthesizer (DDS), but the circuit also includes several filtering stages and a constant reference crystal. The function generator's frequency can be adjusted by the Digital Signal Processor (DSP) via a SPI serial communication interface. Specifications for this circuit include frequency adjustment from 10 to $20 \mathrm{MHz}$, with a resolution of less than $10 \mathrm{~Hz}$ and an accuracy of $100 \mathrm{ppm}$.

\section{B. Tunable Reactive Resonant (RLC) Circuit}

This circuit consists of a parallel RLC circuit (figure 2) where the inductive component is the induction coil; the primary capacitive component is a set of varactors which allow for remote capacitance adjustment via a serial output from the DSP; and a resistor which lowers the Q-value of the reactive components allowing the entire circuit to be held at resonance more easily. This circuit is driven by the function generator through a sense resistor allowing for measurements of voltage across and current through the RLC circuit. Placing a conductive object in front of the coil will cause an amplitude change in the voltage across the RLC circuit and a phase shift between voltage and current outputs indicating that the circuit is no longer at resonance. At this point, either the frequency of the function generator or the capacitance of the varactors can be adjusted by the DSP to return the RLC circuit to resonance. From the change in voltage amplitude and the current through the RLC circuit when a conductive object is placed near the coil, the impedance change of the coil at resonance can be accurately calculated.

\section{RLC Voltage Output Peak Hold Circuit}

This circuit takes the voltage output from the RLC circuit and allows for the measurement of the maximum voltage over a period of time. The time period is controlled by a reset signal from the DSP. At the heart of this circuit is a Texas Instruments TLV3501 high speed voltage comparator which is configured to monitor and maintain the maximum voltage. The output of this circuit, the maximum voltage since the last reset, is sent to the DSP via an analog to digital converter and a SPI serial interface.

\section{RLC Current Output Peak Hold Circuit}

Note that the current output from the RLC circuit is actually a voltage that can be used to calculate current since the voltage is across a constant sense resistor of known value. This circuit takes the current output voltage from the RLC circuit and allows for the measurement of the maximum current over a period of time. The time period is controlled by a reset signal from the DSP. At the heart of this circuit is another Texas Instruments TLV3501 high speed voltage comparator which is configured to monitor and maintain the maximum voltage. The output of this circuit, the maximum current since the last reset, is sent to the DSP via another analog to digital converter and a SPI serial interface.

\section{E. 90 Degree Phase Shift Circuit}

This circuit utilizes a unity gain amplifier in a Sallen-Key configuration to implement a 90 degree phase shift. The phase shift is necessary for the phase detection circuit due to the fact that the phase detector will be unstable when the two inputs are in phase with each other; i.e. the RLC circuit is at resonance. Therefore the output of this circuit, which is the voltage output of the RLC circuit shifted by 90 degrees, is used as one of the inputs to the phase detection circuit.

\section{F. Phase Detection Circuit}

This circuit compares the phase of the current output and the phase-shifted voltage output of the RLC circuit to determine the position of the RLC circuit with respect to resonance. If the output of this circuit indicates that the voltage leads the current by 90 degrees, we know that the RLC is exactly at resonance. At the center of this circuit is the Analog Devices AD8302 Phase/Gain comparator, which accepts the two inputs and provides a DC voltage output representing phase difference. As stated above, this circuit will be unstable when the two inputs are in phase with each other; therefore we avoid this situation by applying the 90 degree phase shift to the voltage input. The DC voltage output of this circuit is converted to a digital value and is sent to the DSP via a SPI serial interface.

\section{G. Digital Signal Processor (DSP)}

This is the main processing unit of the overall circuit. The DSP accepts inputs from the voltage peak hold circuit, the current peak hold circuit, and the phase detection circuit and sets outputs to control the function generator's frequency and the RLC circuit's capacitive component. The DSP also interfaces with the Bluetooth interface to allow control from and provide data to the user interface software residing on a remote PC. The Digital Signal Processor selected for this circuit is the TMS320F2808 manufactured by Texas Instruments. It was chosen primarily because it provides the appropriate number of high speed SPI interfaces, includes substantial on board memory to hold both the control firmware and the necessary data, and can operate at a high enough speed to accommodate design specifications.

\section{H. Bluetooth Wireless Communication Module}

Since a wireless interface with a data collection and control application on a remote PC was desired, the BlueGiga WT12 Bluetooth module was implemented. This module allows two-way serial communications between the remote PC and the DSP. The capabilities of the WT12 module include Bluetooth 2.0 with enhanced data rate to accommodate high 
speed data communication with reduced power consumption over other Bluetooth modules.

\section{RESUlts}

Using portable electronics described previously, and developed in collaboration with Plexus Services Corporation, we measured sensor admittance while the resonant coil is placed into close proximity with a series of standard Potassium Chloride $(\mathrm{KCl})$ salt solutions. Electrical conductivities of $\mathrm{KCl}$ solutions were obtained using data from [21] and corrected for temperature as necessary. Strictly speaking, these values are applicable only to lower frequencies $(<0.1 \mathrm{MHz})$, but presumed accurate at $12.5 \mathrm{MHz}$. $\mathrm{KCl}$ solutions were placed in 0.5 liter plastic bottles having a $7 \mathrm{cmX} 8 \mathrm{~cm}$ rectangular base and $10 \mathrm{~cm}$ height, facilitating full contact with the coil at the side wall. A typical calibration curve is shown in figure 5 , taken at $12.5 \mathrm{MHz}$, which illustrates excellent agreement with the simple form of equation (4). Some of these bottles were sacrificed to measure wall thickness, which was found to vary by about $10 \%$, accounting for the noise that is observed in the figure.

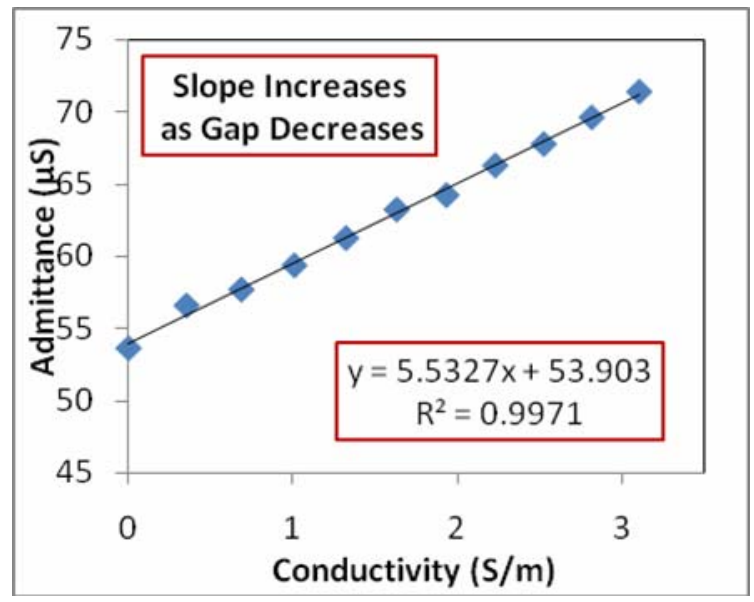

Fig.5 Admittance (micro Siemens) vs. aqueous $\mathrm{KCl}$ Conductivity at a spacing of $1.7 \mathrm{~mm}$.

Plastic spacers were also prepared, allowing us to make additional complete sets of measurements at a variety of separations between coil and specimen ( $\mathrm{KCl}$ solution). As indicated in figure 5, slope is expected to increase as gap distance between coil and specimen decreases. Examination of data at a variety of spacing values led us to a general equation consistent with the physics of equation (4), but also accounting for coil-specimen gap distance, $x$ :

$$
A=A_{0}+\frac{\alpha \sigma}{\beta+x}
$$

$$
\alpha^{\prime}=\frac{\alpha}{\beta+x} ; A_{0}=\frac{1}{R_{p}}+\frac{R_{L}}{\omega^{2} L^{2}} \cong \frac{1}{R_{p}}
$$

Rearranging (5) to the form of (6) below allowed us to test the role played by coil-specimen gap distance:

$$
\frac{\sigma}{A-A_{0}}=\frac{\beta}{\alpha}+\frac{x}{\alpha}
$$

Figure 6 vindicates the form of (6), which was further supported by simulations of the effect of gap distance between coil and specimen using an electromagnetic method of moments package (FEKO) [17]. Once the calibration parameters, $\alpha$ and $\beta$, were found, the sensor was used for invivo human tissue measurements. A helpful aspect of (6) is that while $\beta$ is typically about $3 \mathrm{~mm}$, the spacing used for invivo testing is about $0.1 \mathrm{~mm}$, making precise knowledge of spacing relatively unimportant. In all in-vivo testing thus far, a $0.1 \mathrm{~mm}$ adhesive plastic film was placed over the coil for hygienic considerations. Obviously, no electrical contact is made with the specimen with such an arrangement.

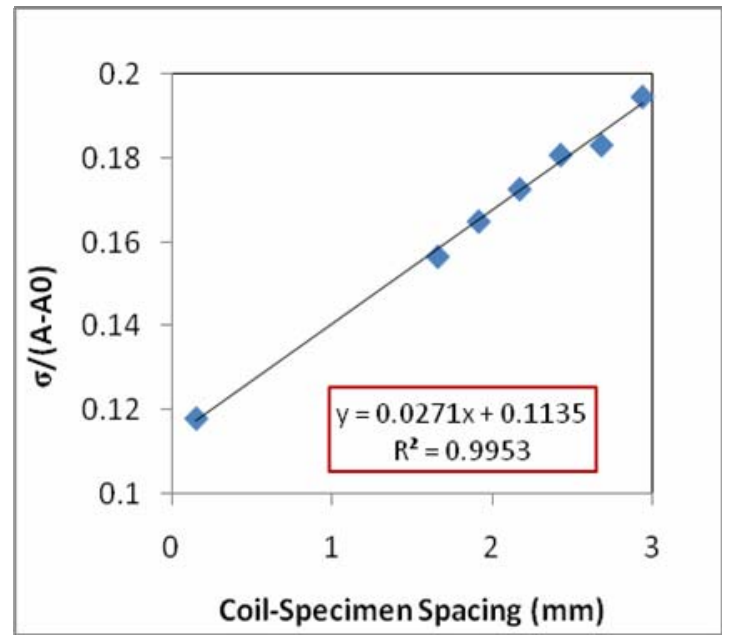

Fig.6 Effect of gap distance. Data collected for 2.0\% Potassium Chloride, having conductivity $=3.11 \mathrm{~S} / \mathrm{m}(23.4 \mathrm{C})$.

\section{Relation of Eddy Current Measurements to Tissue Micro- Structure:}

In order to make sense of the inevitable variation in electrical conductivity that occurs from site to site on the body, and more importantly from one individual to the next, we have developed a simple hypothesis that turns out to be consistent with data collected thus far in two clinical trials. The central idea we use to interpret data considers soft tissue beneath the epidermis as a two-phase medium consisting of low-conductivity micro-vascular tissue and highly conductive 
interstitial fluids. Representation of soft human tissue as two phases of interacting incompressible material is common (Leiderman et. al., [13]). Furthermore, recent research supports the notion that small blood vessels are relatively nonconducting (Stinstra et. al., [14]) in comparison with interstitial fluids. They attribute low conductivity to the insulating nature of the capillary wall and the tendency for non-conductive blood cells to greatly increase the path length for charge carriers. Further discussion of the insulating nature of small vessels is taken up in the appendix.

Since our coil radius is about $1.75 \mathrm{~cm}$, limiting penetration depth to about $1.5 \mathrm{~cm}$, vascular tissue probed by induction coil conductivity sensor (ICCS) technology is believed to consist primarily of very small vessels, such as arterioles, capillaries, venules and lymph vessels - though arteries and veins will contribute if located in the coil's "field of view". The distribution of blood across the various vessel types would of course be dependent upon body location. Because of their interconnected arrangement, flow obstructions through one portion of the vascular circuit will likely have impact on flow elsewhere.

According to our hypothesis, any stimulus, whether thermal, mechanical or physiological, that would cause the volume fraction of the "vascular tissue phase" to decrease, should cause conductivity to increase. For example, deliberately lowering blood pressure in an extremity through increased elevation should cause conductivity to increase. Prior to clinical work, this was tested on one of the authors using our ICCS prototype. As figure 7 clearly shows, electrical conductivity increases in the right forearm after being held vertically above the heart for 60 seconds, providing support for our hypothesis. Furthermore, right and left forearms show statistically significant differences in electrical conductivities - the vascular tree is expected to show differences from right to left, even if only a result of right and left blood pressure differences. For this reason, blood pressures were routinely measured in our clinical studies thus far. The role of blood pressure in determining effect of elevation on conductivity is discussed more fully in a companion paper focused entirely on clinical use of ICCS.

\section{DiscusSION/CONCLUSIONS}

Due to the heterogeneous construction of human tissues and the non-uniform nature of induction fields, the electrical conductivity measured by an induction coil of the type described can only be regarded as an average. But this would have to be true of all in-vivo measurements, whether using contacting electrodes or not. Most human tissue conductivity values reported in the literature are for dead tissues, using setups that involve platinum electrodes making direct electrical contact in geometrically simplified cells. According to Stoy et al [15], electrical property changes for dead tissues measured within 24 hours of death are not believed to be significant to the measurement of conductivity. It is not clear though whether true in-vivo measurement would lead to the same results or that precipitous changes would have occurred at the moment of death - for example, dead tissues do not have the benefit of flowing blood or other physiological conditions associated with living tissues such as active cellular metabolism.

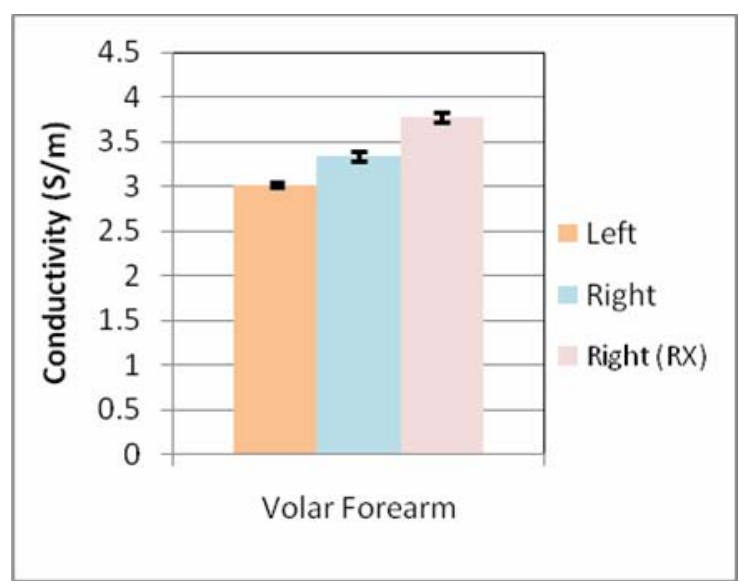

Fig.7 Subject's proximal volar forearms; $\mathrm{RX}=$ raised for 60 seconds.

Conductivity values reported by Stoy et al [15] typically fall in a range from about 0.5 to $1.0 \mathrm{~S} / \mathrm{m}$ for a variety of tissues at either $25{ }^{0} \mathrm{C}$ or $37{ }^{0} \mathrm{C}$. Relative permittivity values are reported to range from about 160 to 500 for several tissues when frequency is about $10 \mathrm{MHz}$, which are much higher than that for water, or even aqueous solutions of simple amino acids. They also report, as do others (C. Gabriel et al [16]), extraordinarily high relative permittivity values $(>1000)$ when frequency falls below about $1 \mathrm{MHz}$, certainly a result of ion accumulation at the platinum electrode interface. The latter effect plays no possible role in an eddy current approach to measuring conductivity since there is no electrode interface, underscoring the inherent difference in the two measurement techniques, with the expectation that results may differ.

To that point, we note that the simple forearm conductivity measurements we report are nominally above $3.0 \mathrm{~S} / \mathrm{m}$, which are higher than those reported in the literature using electrodecontact methods by about a factor of three. We attribute this difference more to the method of measurement than the living status of tissues tested. Whether we use the PLL approach for measuring coil impedance or the swept capacitance method, the results are identical. A valid concern may be raised regarding the tightness of phase lock, which could produce unrealistically high conductivities if not well controlled. In all clinical work thus far [19], phase angle lock was maintained to within $0.37 \pm 0.12$ degrees, regardless of body location. Clearly, no errors were incurred due to inadequate control of phase lock. Another concern we have yet to resolve is the extent to which the coil, when in contact with the body, forms a sufficiently extended system that it weakly couples with 
other circuits of our ICCS prototype. If so, such coupling could insert some additional 'stray' resistance into $\mathrm{R}_{\mathrm{s}}$ of the equivalent circuit shown in figure 2 . The result would be somewhat elevated conductivity readings. In that case, the values we report here would still be proportional to the true conductivity, but they could not be counted upon to represent the true conductivity at $12.5 \mathrm{MHz}$.

\section{ACKNOWLEDGMENT}

The authors wish to acknowledge the contributions of Plexus Services Corporation, in particular for their efforts to develop the prototype induction coil conductivity device used in our studies.

\section{APPENDIX - VESSEL CONDUCTIVITY}

The proposed nonconductive nature of small blood vessels may at first seem puzzling given that the blood flowing within is conductive. However, the conductive blood itself is "fully wrapped" in an insulating sheath that prohibits transverse ionic conduction. Thinking of the characteristics of the induction field, ion currents move along closed loops or pathways that lie entirely inside the soft tissue being probed. Rather than pass through an insulating capillary wall, the current loop is deflected around a capillary when approached. The only way that blood inside of a vessel could contribute to an eddy current is through passage of the current through the vessel wall at one point and then emergence elsewhere. This is not expected to easily happen, so that blood vessels introduce barriers to electrical current flow, forcing the current to take a "detour". This is the basis of crack and fissure detection in otherwise conductive media by the eddy current method. A review of models used to support crack detection via the eddy current approach is given in [18].

\section{REFERENCES}

[1] Haemmerich, D., Staelin, S.T., Tsai, J.Z. (2003). In vivo electrical conductivity of hepatic tumors. Physiol. Meas., 24, 251-260.

[2] Holder, D.S. (ed.) (2004). Electrical Impedance Tomography: Methods, History and Applications. Institute of Physics.

[3] Arnold, D., Meeten, G.H. (1988). Frequency measurements in the inductive measurement of electrical conductivity. J. Phys. E : Sci. Instrum., 21, 448-453.

[4] Guardo, R., Charron, G., Goussard, Y., Savard, P. (1997). Contactless measurement of thoracic conductivity changes by magnetic induction. In Proc. $19^{\text {th }}$ Int. Conf. IEEE/EMBS, October 30, 2450-2453.

[5] Netz, J., Forner, E., Haagemann, S. (1993). Contactless impedance measurement by magnetic induction - a possible method for investigation of brain impedance. Physiol. Meas., 14, 463-471.
[6] Hart, L.W., Ko, H.W., Meyer, J.H., Vasholz, D.P., Joseph, R.I. (1988). A noninvasive electromagnetic conductivity sensor for biomedical applications. IEEE Trans. on Biomed. Eng., 35 (12), 1011-1022.

[7] Schnyders, H.S., Saboungi, M.L., Enderby, J.E. (1999). Noninvasive simultaneous determination of conductivity and permeability. Appl. Phys. Lett., 75 (20), 3213-3215.

[8] Probst, P.A., Collet, B., MacInnes, W.W. (1976). Marginal oscillator optimized for radio-frequency size effect measurements. Rev. Sci. Instrum., 47 (12), 15221526.

[9] Harpen, M.D. (1988). Distributed self-capacitance of magnetic resonance surface coils. Phys. Med. Biol., 33 (9), 1007-1016.

[10] Harpen, M.D. (1988). Influence of skin depth on NMR coil impedance; Part II. Phys. Med. Biol., 33 (5), 597605.

[11] Zaman, A.J.M, Long, S.A., Gardner, C.G. (1980). The impedance of a single-turn coil near a conducting halfspace. J. Nondestruct. Eval., 1 (3), 183-189.

[12] Jackson, J.D. (1999). Classical Electrodynamics. New York: J. Wiley \& Sons.

[13] Leiderman, R., Barbone, P.E., Oberai, A.A., Bamber, J.C. (2006). Coupling between elastic strain and interstitial fluid flow: ramifications for poroelastic imaging. Phys. Med. Biol., 51, 6291-6313.

[14] Stinstra, J.G., Shome, S., Hopenfeld, B., Macleod, R.S. (2005). Modeling the passive cardiac electrical conductivity during ischemia. Med. Biol. Comput. Nov., 43 (6), 776-782.

[15] Stoy, R.D., Foster, K.R., Schwan, H.P. (1982). Dielectric properties of mammalian tissues from 0.1 to $100 \mathrm{MHz}$ : a summary of recent data. Phys. Med. Biol., 27 (4), 501513.

[16] Gabriel, C., Gabriel, S., Corthout, E. (1996). The dielectric properties of biological tissues: I. Literature survey. Phys. Med. Biol., 41, 2231-2249.

[17] FEKO, EM Software and Systems. Retrieved from http://www.feko.info/

[18] Knopp, J.S., Aldrin, J.C., Jata, K.V. (2009). Computational methods in eddy current crack detection at fastener sites in multi-layered structures. Nondestruct. Test. Evaluat., 24 (1\& 2), 103-120.

[19] Feldkamp, J.R., Heller, J. (2009). Effects of extremity elevation and health factors on soft tissue electrical conductivity. Meas. Sci. Rev., 9, No. 6, 169-178.

[20] Dean Straw, R. (ed.) (2006). The ARRL Handbook for Radio Communications 2006 : $83^{\text {rd }}$ Edition. Newington, CT: ARRL, 6.12.

[21] Campbell, A.N., Kartzmark, E.M. (1955). The electrical conductance of strong electrolytes: a test of stokes equation. Canadian J. of Chem., 33, 887-894. 